\title{
Optimization of Exposure Conditions for Computed Radiology Exams in Neonatal Intensive Care
}

\author{
Elisa Rizzi ${ }^{*}$, Silvia Emanuelli1, Simonetta Amerio', Diego Fagan², \\ Francesca Mastrogiacomo ${ }^{2}$, Paola Gianino3 ${ }^{3}$, Federico Cesarani ${ }^{4}$ \\ ${ }^{1}$ Department of Health Physics, Cardinal Guglielmo Massaia Hospital, Asti, Italy \\ ${ }^{2}$ Radiology Institute of University, Città della Salute e della Scienza, Torino, Italy \\ ${ }^{3}$ Pediatrics Division, Cardinal Guglielmo Massaia Hospital, Asti, Italy \\ ${ }^{4}$ Radiology Division, Cardinal Guglielmo Massaia Hospital, Asti, Italy \\ Email: erizzi@asl.at.it
}

Received 22 November 2013; revised 22 December 2013; accepted 30 December 2013

Copyright (C) 2014 by authors and Scientific Research Publishing Inc.

This work is licensed under the Creative Commons Attribution International License (CC BY). http://creativecommons.org/licenses/by/4.0/ (c) (i) Open Access

\begin{abstract}
This paper performs a review of existing literature about neonatal imaging in intensive care; we notice that the multiplicity of approaches results in different and sometimes conflicting solutions to optimize acquisition technique of X-ray images. European Guidelines still refer to screen-film combinations used in past decades, current usage of digital technology requires an additional effort to reduce dose to infants and to optimize the sensor's response exploiting their properties. In this work we investigate response changes of digital medium (computed radiography plates), due to alterations of the beam through incubators components. All combinations in use in our Hospital were tested for evaluating dosimetry and image quality and new exposure solutions were devised to optimize radiology exams, taking into account solutions suggested by the equipments makers. Dose measured was compared with dose levels suggested by European Guidelines, evaluating radiation-induced risk too. Image quality was evaluated in a double-blind comparison by radiologists. An easily repeatable optimization procedure is proposed intended to reduce delivered dose well below European guidelines. The proposed study allowed us to instruct the technologists on the most appropriate methodology for performing the radiology exam, by standardizing the approach to Neonatal Intensive Care Units. We have demonstrated also to radiologic technologists reluctant to use the $\mathrm{X}$-ray tray, as it may optimize imaging in the incubator. We were also able to reduce dose-and radiation-induced risk too-of $37 \%-67 \%$ depending on the previously used operating mode.
\end{abstract}

*Corresponding author. 
Keywords

Neonatal Intensive Care Units; Dose Reduction; Image Quality; Computed Radiography

\section{Introduction}

Digital imaging technology led to the necessity of revising and optimizing delivered dose levels in radiodiagnostics, and to the birth of new issues related to the physical properties of digital equipment. A correct optimization process should start with the correct selection of beam quality [1] to obtain best imaging, and then proceed with a choice of parameters to minimize patient delivered dose.

One of the areas in which these issues have a significant and immediate effect is neonatal imaging, especially when premature infants health conditions require several X-ray exposures in the first weeks of life [2], [3].

Exposures on infants in incubators in intensive care (Neonatal Intensive Care Units-NICU) raise questions on possible modifications to the response of the imaging supports currently in use (in our case, Computed Radiography plates or CR plates) due to possible alterations occurring on radiation beam when passing through the various components of a NICU.

Each radiologic technologist, when performing the required exam, has a large array of possible operating choices for neonatal intensive care, all of them methodologically sound but with a direct impact on image quality and imparted dose. The traditional method of performing the exam involves the positioning of the radiology cassette (digital or film) directly below the infant [4], [5]: this leads to an image without any enlargement, allows visual check of collimation by means of the contrast provided by the black background of the radiology cassettes and does not cause additional attenuation. The method generally suggested by operators in neonatal care [6], on the other hand, stems from considering that the incubator is meant to provide a stable and protective environment for premature babies, and thus requires the usage of the potter to minimize unnecessary movements and disturbance to the infant; images thus obtained are affected by an enlargement factor which makes judgement of collimation more difficult, and the radiation beam must pass through the extra thickness of the mattress and of the cassette-holder system, possibly incurring in attenuation and alteration of the energy spectrum.

A further variable is the presence or removal of the NICU canopy; typically it is left in place, but this provides a further cause of modification of the radiation beam.

Choice of exposure parameters and their optimization is of utmost importance in this practice, since patients are premature infants who often require several exposure to ionizing radiations in their first weeks of life; radiosensitivity in children is higher than in adults since their organs are nearer to the surface and still in development, and they have a higher probability of stochastic long-term effects due to their longer life expectancy: it is thus important to optimize radiology practice and to evaluate related radiation-induced risk [2], [7], [8].

International Committees on Radioprotection [9]-[13], while remarking the sensitivity of paediatric exams, don't have a systematic approach to exposures of infants requiring usage of NICU, and as a consequence the "good technique" recommendations on this topic are quite scarce. In addition to this, European guidelines and the subsequent laws issued by Countries of the EU are quite old and still related to screen-film systems, while almost everywhere Computed Radiography or Direct Radiography systems are now in use. Exposure conditions suggested by EUR16261 [13] (i.e. addition of filtration) might no longer be optimal due to the different response curve of the new imaging systems, and the same could be said of the maximum suggested dose limits (Reference Diagnostic Levels, RDL) which could be revised and maybe lowered [9], [13], [14].

An analysis of literature on exposure of infants in intensive care [2]-[6], [15]-[25], leads to contrasting conclusions for the choice of optimal exposure parameters and positioning of image device. Concerning the selection of beam quality, some studies [3], [15], [16] advise, in agreement to what indicated in [13], the usage of additional filtering at the output of the $\mathrm{X}$-ray beam: the purpose is sharpening the beam by removing low energy components which don't have a contribution on image formation and actually increase noise while increasing imparted dose. In some studies [4], [17], [20], [21], though, usage of additional filtering is not used. In [22] the additional aluminum filtration is encouraged provided that you increase the $\mathrm{kV}$, while copper filtration is not recommended.

Duggan et al. [24] recommend using hafnium and aluminum filter employing higher kV than [13]. 
Right exposure parameters selection is well debated in [5] and the wide variability about exposure parameters (and skin doses) in literature is summarized in [21], [23].

The problem is also imaging device related: although the transition to digital imaging is accomplished by several years, there are few papers on it within NICU [16], [20], [26].

Finally also the positioning of the imaging device is still debated between under the baby [4], [5] and into cassette tray of the NICU [6].

This work aims to study the impact on image quality obtained by the several possible operating configurations, to establish the optimal one related to CR imaging. To this purpose, different operating solutions available to Radiologic Technologists have been evaluated and compared in terms of Entrance Skin Dose (ESD) to the patient and related risk factors, but also in terms of image quality.

A further objective was optimizing exposure parameters employed in our hospital: to this purpose, new operating solutions and radiological parameters were tested, taking inspiration from good technique recommendations and literature on topic. Dose levels for each configuration have been compared with RDLs suggested by European Guidelines and enforced by current Italian law, and radiation-induced risk has been evaluated; this approach did not forget image quality, always evaluated double-blind by three Radiologists.

\section{Methods and Materials}

Radiological exposures at the Divisione Nido Patologico/Reparto Pediatria of Ospedale Cardinal Massaia ASL AT, where 6 intensive neonatal Caleo units by Drager are operational, have been performed with X-ray portable Philips Practix 160 equipment.

Imaging system consists of Computed Radiography CR Agfa MD 4.0 high resolution plates, made with a non-structured (powder) single-layer of BaSrFBrI:Eu phosphors. The CR Agfa 75.0 reader was equipped with standard and advanced image processing capabilities including automatic collimation masking, window and level adjustments, gray-scale processing, and advanced spatial frequency processing including Multi-Scale Image Contrast Amplification (MUSICA). The exposed CR cassettes are transported to the CR reader and processed according to the manufacturer's paediatric image processing protocol which provides 100-speed class specification; in the last section of the study, we altered this setting to check the results.

Diagnostic images have been evaluated by Radiologists on dedicated "30-inch" Barco Coronis Fusion 6MP MDCC-6130 displays, used as two seamless 3 Megapixel heads. The monitors were calibrated to the DICOM grayscale standard display function.

The entire process for image gathering and visualization was optimized and regularly monitored with periodical quality controls by the Medical Physics Experts.

Incubators are equipped by accessories such as sensors for controlling temperature and oxygen, but in this work only equipment with a possible effect on attenuation was taken into account: cassette-holder, removable polycarbonate canopy, and polyurethane-polyester mattress (size $45.5 \times 61.5 \times 3 \mathrm{~cm}$ ) with encasing of styrol butadiene thermal foam. Incubators height (ground to mattress surface) is settable from a minimum of $800 \mathrm{~mm}$ up to a maximum of $1100 \mathrm{~mm}$.

Radiation beam must pass through the polycarbonate canopy and, when the cassette-holder is used, the mattress and its support too. An enquiry in our hospital showed that, whenever possible, some Technologists prefer to open the canopy and many of them are in doubt whether to use the cassette-holder or not. Removal of the canopy is preferred by some to avoid introducing attenuation of unknown amount, while on the other hand usage of the potter raises questions of a more practical nature: the risk could be obtaining images which are too enlarged or making collimation on the patient harder, because the black background of the cassette offers a better contrast than the light-colored background of mattress and sheets.

\subsection{Phase 1: Study of Currently Adopted Exam Operating Conditions}

Radiological exams performed with CRs do not allow writing in Dicom headers of any information on exposure parameters, so a survey among Radiologic Technologists was necessary to define the average exposure parameters and the modalities used for performing the exams.

This preliminary survey showed good uniformity on chosen exposure parameters, however, is difficult to determine the focus to patient distance (FPD) used because of operational difficulties related to its measurement. To try to reduce delivered dose, all operators agree in placing the $\mathrm{X}$-ray tube at its higher excursion limit, few of 
them change and/or measure the incubator's height from the floor.

It was decided to conduct the study in terms of maximum and minimum delivered dose to the patient, or working with the X-ray tube at its maximum height and the incubator at the maximum (FPDmin $=80 \mathrm{~cm}$ ) and minimum $($ FPDmax $=110 \mathrm{~cm})$ of his excursion.

\subsection{Phase 2: Evaluation of Methodologies Currently in Use for Performing the Exam: Dosimetry and Image Quality}

To assess the entity of possible attenuation of the radiation beam when passing through the different materials of the incubator, measurements have been performed to determine separate attenuation factors of canopy, mattress and relative support, and of the combination of all these items (canopy + mattress with support). Measurements were performed with Radcal 10X5-6 ionization chamber and dedicated Radcal 9010 electrometer in good geometry conditions.

Since operation with the cassette in its holder seems the most reasonable configuration, enlargement factor in this condition was measured and Radiologists expressed their evaluations.

Standard exposure parameters used in practice being known, we measured the ESD by simulating the infant's body with a polymethacrylate (PMMA) $5 \mathrm{~cm}$ thick phantom placed inside the incubator, and by positioning the ionization chamber above and in direct contact with it. The obtained measurement is already inclusive of back scattered radiation, according to definition of ESD [27]; since FPD used Technologists in clinical routine was not known, evaluations were performed at $\mathrm{FPD}_{\text {min }}$ and $\mathrm{FPD}_{\max }$.

Induced tumours risk was then evaluated by means of the definition provided by International Commission on Radiological Protection (ICRP) [7]:

$$
R=R F \cdot E=R F \cdot \sum_{T} w_{T} \cdot D_{T}
$$

where $R F$ is the risk factor conservatively estimated as $13 * 10^{-2} \mathrm{~Sv}^{-1}$ [7], $E$ is the effective dose associated to each exposure, $w_{T}$ is the weight factor of tissue or organ exposed to radiation, and $D_{T}$ is the average dose absorbed by it.

For estimating effective dose (and organ dose), the PCXMC [28] software based on Montecarlo code was used: it allows to simulate the interaction of ionizing radiation with the patient, by varying beam spectrum and kind of patient (from infant to adult).

The program requires to provide the following inputs: size of radiation field, its position according to the anatomic region of interest (by means of a graphical tool, too), the kind of patient (in this case, newborn), the energy spectrum of beam and Entrance Surface Air Kerma (ESAK). This last value (ESAK) was calculated by measuring the radiation's output in different operating conditions, according to formula:

$$
\mathrm{ESAK}=R(\mathrm{kV}) \cdot \mathrm{mAs} \cdot d^{2}
$$

where $R(\mathrm{kV})$ is the output $[\mu \mathrm{Gy} / \mathrm{mAs}]$ of the equipment at a distance of 1 meter at the employed voltage, $\mathrm{mAs}$ is the product of set current and time, $d$ is the distance in meters between focus and patient skin.

The output of the program is a report listing the evaluation of dose to organs for 29 organs and tissues, and the related effective dose calculated according to the new weighting factors from issue 103 of International Commission on Radiological Protection [29]; all dose estimations are provided with their statistical confidence.

Image quality was evaluated by means of a LEEDS TOR CDR geometrical phantom and a step wedge phantom. The first phantom is a Perspex disk with several inserts: a variable frequencies (0.5 to $14.3 \mathrm{lp} / \mathrm{mm})$ linepairs to allow evaluation of spatial resolution, a group of 17 large diameter $(11 \mathrm{~mm})$ disks with variable contrast (from 0.076 to 0.0022 ) to allow evaluation of detection threshold for large detail at low contrast, and a group of 17 small diameter $(0.5 \mathrm{~mm})$ disks to evaluate detection of small detail. This last group of small disks allows to perform a test which is very sensitive to image noise. The wedge phantom is made of 21 aluminium steps and allows evaluation of grayscale levels and dynamic range.

Phantoms were positioned above the PMMA, and CRs alternately directly below the PMMA or in the cassette-holder. Standard exposure parameters used by Technologists were set, and images were gathered in all possible combinations of operating conditions: open or closed canopy, FPD. Each image was tagged with an ID code known only to Medical Physicist, and then sent to the PACS for double-blind evaluation by three Radiolo- 
gists.

\subsection{Phase 3: Standardization and Optimization of Exam Performance Technique}

In this phase, the most appropriate operating modalities were determined and, with this set-up, the second objective of this work was faced: optimization of exposure parameters.

Working conditions as close as possible to EUR 16261 suggestions were adopted, using additional filtration and trying to work at higher $\mathrm{kV}$ but lowering $\mathrm{mAs}$; in this phase, default paediatric protocols factory-stored in the tubes equipment were tested too: they indicate a suggested focus-to-CR distance of $100 \mathrm{~cm}$ (not too dissimilar from the $93 \mathrm{~cm}$ that we will choose) and are subdivided for infant weight categories. In each condition ESD, image quality and radiation-induced risk were estimated.

In Table 1(a) (columns 1 and 2) all different exposure conditions are shown: mode 1 employs the average exposure parameters set by Technologists; in 1-A a $4 \mathrm{~mm}$ aluminium filtration (equivalent to $1 \mathrm{~mm} \mathrm{Al}+0.1 \mathrm{~mm}$ $\mathrm{Cu})$ was added; mode 2 employs exposure parameters suggested by EUR16261; in 2-A the same parameters were used but with the filter removed; modes indicated with $\mathrm{P}$ are the ones factory-stored in the Philips equipment, with the number indicating the weight in $\mathrm{kg}$ of the infant.

In the last phase of the study, we tried to optimize CR processing: usually Technologists process plates with the protocol suggested by Agfa for paediatric exams, using a speed class of 100. We wondered if this was really the optimal value.

Speed class 100 is usually employed for areas with low contrast (such as mammography), 200 is usually employed for thorax areas in adult patients, while 400 is used for enhancing radiologic contrast in exams with radiocontrast agents. As a consequence, we replicated the image quality evaluations by setting speed class 200 and 400 too.

\section{Results and Discussion}

\subsection{Phase 1: Study of Currently Adopted Exam Operating Conditions}

From the survey among Technologists, we noticed that usually diagnostic images are taken by setting a tube voltage of $55 \mathrm{kV}$ and a current of $3.2 \mathrm{mAs}$, for a resulting exposure time of $18 \mathrm{~ms}$.

Focus-to-patient skin distance was not determined, since all Technologists position the tube at its maximum height but neglect to measure the height of NICU from ground level. To standardize operating procedure, image quality evaluations were thus performed in conditions of maximum and minimum ESD, that is with the unit at the maximum $\left(\mathrm{FPD}_{\min }=80 \mathrm{~cm}\right)$ and minimum $\left(\mathrm{FPD}_{\max }=110 \mathrm{~cm}\right)$ of its possible excursion.

\subsection{Phase 2: Evaluation of Methodologies Currently in Use for Performing the Exam: Dosimetry and Image Quality}

Attenuation of the radiation beam when passing through the various components of the NICU was evaluated as first step; the attenuation of the canopy was established at $17 \%$, while the mattress and its support cause an attenuation of approximately 15\%. For images with the CR in the cassette-holder, the enlargement factor was measured at 1.09, a value considered negligible for diagnostic purposes by Radiologists.

Again by setting standard exposure parameters used in clinical practice, image quality was evaluated for all standard operating conditions.

In Tables 2(a) and (b) results of evaluations by three Radiologists are shown: for sensitivity to low contrast and small detail the number of detected inserts is listed; for spatial resolution the number of lines/mm of the finest detected target is listed.

The three evaluators show a good degree of agreement: the differences are in the range of \pm 1 inserts, which is the sensitivity of the measurement. Average evaluation values show how image quality seems to be consistent in all configurations, with differences again in the \pm 1 inserts range: in particular, images obtained at FPD $80 \mathrm{~cm}$ and at FPD $110 \mathrm{~cm}$ with CR positioned directly under the phantom seem to be substantially equivalent in all set up. A slight degradation of detail separation at low contrast can be noticed, however, with closed canopy and CR in the potter at FPD $110 \mathrm{~cm}$.

Using the NICU in a configuration meant not to disturb the infant would then seem ideal, and as a conesquence the preferred operating mode would then be at $80 \mathrm{~cm}$ FPD, closed canopy and cassette inside the potter. To 
Table 1. (a) Optimization study of exposure parameters: image quality evaluation; (b) optimization study of exposure parameters: entrance skin dose, effective dose and radioinduced risk evaluation.

(a)

\begin{tabular}{|c|c|c|c|c|c|}
\hline $\begin{array}{l}\text { Identify } \\
\text { code }\end{array}$ & RX setup & $\begin{array}{c}\mathrm{N}^{\circ} \text { of low contrast inserts } \\
\text { detected }\end{array}$ & $\begin{array}{l}\mathrm{N}^{\circ} \text { of small detail } \\
\text { Inserts detected }\end{array}$ & $\begin{array}{l}\text { Spatial resolution } \\
\text { detected }\end{array}$ & Dynamic range \\
\hline 1 & $55 \mathrm{kV} 3.2 \mathrm{mAs}$ & 11 & 14 & $3.15 \mathrm{lp} / \mathrm{mm}$ & 8 \\
\hline $1-\mathrm{A}$ & 55 kV 3.2 mAs; 4 mmAl & 9 & 11 & $2.5 \mathrm{lp} / \mathrm{mm}$ & 8 \\
\hline 2 & 60 kV 1 mAs; 4 mmAl & 7 & 10 & $2.5 \mathrm{lp} / \mathrm{mm}$ & 7 \\
\hline 2-A & $60 \mathrm{kV} 1 \mathrm{mAs}$ & 11 & 12 & $2.8 \mathrm{lp} / \mathrm{mm}$ & 8 \\
\hline $\mathrm{P} 2.2$ & $62 \mathrm{kV} 0.4 \mathrm{mAs}$ & 9 & 9 & $2.5 \mathrm{lp} / \mathrm{mm}$ & 7 \\
\hline P1.8 & $60 \mathrm{kV} 0.4 \mathrm{mAs}$ & 8 & 9 & $2.24 \mathrm{lp} / \mathrm{mm}$ & 6 \\
\hline P1.4 & $60 \mathrm{kV} 0.32 \mathrm{mAs}$ & 7 & 8 & $2.24 \mathrm{lp} / \mathrm{mm}$ & 6 \\
\hline P1 & $60 \mathrm{kV} 0.2 \mathrm{mAs}$ & 7 & 7 & $1.4 \mathrm{lp} / \mathrm{mm}$ & 6 \\
\hline
\end{tabular}

(b)

\begin{tabular}{cccc}
\hline RX setup & ESD $^{a}[\mu \mathrm{Gy}]$ & Effective dose $[\mu \mathrm{Sv}]$ & Radio induced risk $\left(\times 10^{-6}\right)$ \\
\hline $55 \mathrm{kV} 3.2 \mathrm{mAs}$ & $88.47 \pm 3.54$ & $47.72 \pm 2.10$ & 6.2 \\
$55 \mathrm{kV} 3.2 \mathrm{mAs} ; 4 \mathrm{mmAl}$ & $29.23 \pm 1.17$ & $19.55 \pm 0.96$ & 2.5 \\
$60 \mathrm{kV} 1 \mathrm{mAs} ; 4 \mathrm{mmAl}$ & $12.40 \pm 0.56$ & $8.72 \pm 0.43$ & 1.1 \\
$60 \mathrm{kV} 1 \mathrm{mAs}$ & $33.80 \pm 1.35$ & $19.26 \pm 0.83$ & 2.5 \\
$62 \mathrm{kV} 0.4 \mathrm{mAs}$ & $17.03 \pm 0.83$ & $9.89 \pm 0.43$ & 1.3 \\
$60 \mathrm{kV} 0.4 \mathrm{mAs}$ & $15.90 \pm 0.72$ & $9.06 \pm 0.39$ & 1.2 \\
$60 \mathrm{kV} \mathrm{0.32} \mathrm{mAs}$ & $9.33 \pm 0.42$ & $5.32 \pm 0.23$ & 0.7 \\
$60 \mathrm{kV} 0.2 \mathrm{mAs}$ & $6.2 \pm 0.29$ & $3.55 \pm 0.15$ & 0.5 \\
\hline
\end{tabular}

a. ESD = Entrance Skin Dose.

Table 2. (a) Comparison of assessments of image quality by three radiologists; (b) Comparison of assessments of image quality by three radiologists.

(a)

\begin{tabular}{|c|c|c|c|c|c|c|c|c|}
\hline Set up & \multicolumn{4}{|c|}{$\mathrm{N}^{\circ}$ of low contrast inserts detected } & \multicolumn{4}{|c|}{$\mathrm{N}^{\circ}$ of small detail inserts detected } \\
\hline $\mathrm{FPD}^{\mathrm{a}}=80 \mathrm{~cm}$ & A & B & $\mathrm{C}$ & $A v^{b}$ & A & $\mathrm{B}$ & $\mathrm{C}$ & $A v^{\mathbf{b}}$ \\
\hline CR below PMMA + open canopy & 13 & 11 & 12 & 12 & 14 & 13 & 14 & 14 \\
\hline CR below PMMA + closed canopy & 12 & 11 & 13 & 12 & 13 & 13 & 13 & 13 \\
\hline CR inside the tray + open canopy & 11 & 11 & 11 & 11 & 13 & 13 & 14 & 13 \\
\hline CR inside the tray + closed canopy & 11 & 11 & 11 & 11 & 13 & 12 & 13 & 13 \\
\hline Set up & \multicolumn{4}{|c|}{$\mathrm{N}^{\circ}$ of low contrast inserts detected } & \multicolumn{4}{|c|}{$\mathrm{N}^{\circ}$ of small detail inserts detected } \\
\hline$F P D^{a}=110 \mathrm{~cm}$ & A & B & $\mathrm{C}$ & $A v^{\mathbf{b}}$ & A & $\mathrm{B}$ & $\mathrm{C}$ & $A v^{b}$ \\
\hline CR below PMMA + open canopy & 12 & 11 & 12 & 12 & 14 & 12 & 14 & 14 \\
\hline CR below PMMA + closed canopy & 12 & 11 & 12 & 12 & 12 & 12 & 12 & 12 \\
\hline CR inside the tray + open canopy & 13 & 12 & 13 & 13 & 14 & 12 & 14 & 14 \\
\hline CR inside the tray + closed canopy & 11 & 10 & 10 & 10 & 13 & 12 & 13 & 13 \\
\hline
\end{tabular}

(b)

\begin{tabular}{|c|c|c|c|c|c|c|c|c|}
\hline Set up & \multicolumn{4}{|c|}{ Spatial resolution detected $(\mathrm{lp} / \mathrm{mm})$} & \multicolumn{4}{|c|}{ Dynamic range discriminate } \\
\hline $\mathrm{FPD}^{\mathrm{a}}=80 \mathrm{~cm}$ & A & $\mathrm{B}$ & $\mathrm{C}$ & $A v^{\mathbf{b}}$ & A & $\mathrm{B}$ & $\mathrm{C}$ & $A v^{b}$ \\
\hline CR below PMMA + open canopy & 3.15 & 2.8 & 3.15 & 3.15 & 11 & 10 & 10 & 10 \\
\hline CR below PMMA + closed canopy & 2.8 & 3.15 & 3.15 & 3.15 & 10 & 10 & 11 & 10 \\
\hline CR inside the tray + open canopy & 2.8 & 3.55 & 3.15 & 3.15 & 8 & 8 & 10 & 8 \\
\hline CR inside the tray + closed canopy & 3.55 & 3.15 & 2.8 & 3.15 & 8 & 8 & 9 & 8 \\
\hline Set up & \multicolumn{4}{|c|}{ Spatial resolution detected (lp/mm) } & \multicolumn{4}{|c|}{ Dynamic range discriminate } \\
\hline$F P D^{a}=110 \mathrm{~cm}$ & A & $\mathrm{B}$ & $\mathrm{C}$ & $A v^{b}$ & A & B & $\mathrm{C}$ & $A v^{b}$ \\
\hline CR below PMMA + open canopy & 2.8 & 3.15 & 3.55 & 3.15 & 10 & 11 & 10 & 10 \\
\hline CR below PMMA + closed canopy & 3.15 & 3.15 & 3.15 & 3.15 & 10 & 11 & 10 & 10 \\
\hline CR inside the tray + open canopy & 3.15 & 3.15 & 3.15 & 3.15 & 9 & 9 & 8 & 9 \\
\hline CR inside the tray + closed canopy & 3.15 & 3.15 & 2.8 & 3.15 & 8 & 9 & 9 & 9 \\
\hline
\end{tabular}


assess variability of absorbed dose, a measurement with closed canopy at the two extreme FPDs was performed, evaluating at the same time the risk of inducing a lethal tumour in the first 10 years of life of the patient [7].

Results are the following:

- $\operatorname{ESD}_{\mathrm{FPD}} 80=(88.47 \pm 3.54) \mu \mathrm{Gy}$;

Radiation induced risk per million patients $=6.3$

- $\operatorname{ESD}_{\mathrm{FPD} 110}=(46.08+1.84) \mu \mathrm{Gy}$;

Radiation induced risk per million patients $=3.3$

As expected, ESD and radiation induced risk can be halved by using $110 \mathrm{~cm}$ FPD, anyway risk remains very low and at comparable values in both configurations.

Measured ESDs must be compared with maximum dose levels recommended by European Commission Guidelines [13] and regulated by Italian laws [14], which is $80 \mu \mathrm{Gy}$, and also with the more recent NRPB [9] which suggests average values lower than $50 \mu \mathrm{Gy}$.

\subsection{Phase 3: Standardization and Optimization of Exam Performance Technique}

Dosimetric and image quality results discussed in phase 2 led to the conclusion that a comparable image quality can be achieved by halving the dose imparted to the patient; it looks like it's possible to further reduce ESD (even at $\mathrm{FPD}_{\max } 110 \mathrm{~cm}$ ) by optimizing exposure parameters, albeit at the price of a small degradation of image quality.

In our attempt to standardize the procedure, we chose to opt for $\mathrm{FPD}_{\min } 80 \mathrm{~cm}$ (focus-to-CR distance $=93 \mathrm{~cm}$ ) as it is compliant to what suggested by European guidelines EUR16261, and to suggest to Technologists the usage of the cassette holder without opening the canopy of the NICU.

The following optimizations have been performed by executing dosimetric and image quality tests in the operating modes listed in Table 1 (a) (columns 1 and 2).

Concerning image quality, in this test and in the following ones the same variability of \pm 1 inserts was reported by the observers. In an attempt to improve readability of results, in Table 1(a) the averages of evaluations by three Radiologists are shown.

Modifications to operating mode 1 led to an ESD always lower than that of mode 1 with $\mathrm{FPD}_{\max }$; notice how, as expected, a decrease in dose leads to a slight degradation of image quality, which becomes noticeably evident in modes P 2.2-P 1.8-P 1.4-P 1.

Dynamic range decreases below $20 \mu \mathrm{Gy}$ of ESD.

The presence of additional filtration seems to degrade the resolution of low contrast and spatial resolution, a consideration which is particularly evident by comparing two operating modes with same absorbed dose such as 1-A and 2-A. Operating modes with best image quality are the ones marked as 1 and 2-A; operating mode 2-A however imparts an ESD which is $40 \%$ lower than 1, it will then be chosen as gold-standard for performing the exams.

In the last part of the study, we then tried to optimize CR processing methodologies: image quality evaluation was repeated for all combinations shown in Table 1(a), processing the plates at 200 and 400 speed class to evaluate the possibility of adopting of a different and better combination of radiologic parameters.

The averages of results thus obtained (Table 3) always show a worsening in detection of inserts at 200 speed, particularly evident in terms of spatial resolution; in particular in modes with imparted ESD lower than $10 \mu \mathrm{Gy}$ image quality becomes noticeably compromised, since with low doses $(<1.5 \mu \mathrm{Gy})$ reaching the plates the increase in noise has a stronger effect on detail separation.

Usage of 400 speed (Table 4) does not affect image quality noticeably: differences in terms of contrast, detail and spatial resolution between images at 100 and 400 speed are always in terms of \pm 1 insert; however, Radiologists prefer images at 100 speed for their higher contrast between blacks and whites.

\section{Conclusions}

Our study allowed us to instruct the Radiologic Technologists on the most appropriate methodology for performing the exam, by standardizing the approach to NICU: in particular, it was proved that for the same set of exposure parameters, the attenuation due to the canopy and the cassette-holder does not degrade image quality noticeably. The enlargement of images due to the positioning of the CR in the cassette-holder has been judged negligible for diagnostic purposes by Radiologists. Technologists will then work from now on without removing 
Table 3. Quality image comparison: Speed Class 100 vs Speed Class 200.

\begin{tabular}{|c|c|c|c|c|c|c|}
\hline \multirow{2}{*}{ RX setup } & \multicolumn{2}{|c|}{$\mathrm{N}^{\circ}$ of low contrast inserts detected } & \multicolumn{2}{|c|}{$\mathrm{N}^{\circ}$ of small detail inserts detected } & \multicolumn{2}{|c|}{ Spatial resolution detected $[\mathrm{lp} / \mathrm{mm}]$} \\
\hline & S 100 & S 200 & S 100 & S 200 & S 100 & S 200 \\
\hline $55 \mathrm{kV} 3.2 \mathrm{mAs}$ & 11 & 12 & 14 & 13 & 3.15 & 2.24 \\
\hline $55 \mathrm{kV} 3.2 \mathrm{mAs} 4 \mathrm{mmAl}$ & 9 & 7 & 11 & 9 & 2.5 & 2.0 \\
\hline $60 \mathrm{kV} 1 \mathrm{mAs} 4 \mathrm{mmAl}$ & 7 & 7 & 10 & 10 & 2.5 & 1.8 \\
\hline $60 \mathrm{kV} 1 \mathrm{mAs}$ & 11 & 9 & 12 & 11 & 2.8 & 1.8 \\
\hline $62 \mathrm{kV} 0.4 \mathrm{mAs}$ & 9 & 6 & 9 & 10 & 2.5 & 1.6 \\
\hline $60 \mathrm{kV} 0.4 \mathrm{mAs}$ & 8 & 6 & 9 & 6 & 2.24 & 1.4 \\
\hline $60 \mathrm{kV} 0.32 \mathrm{mAs}$ & 7 & 4 & 8 & 1 & 2.24 & 1.25 \\
\hline $60 \mathrm{kV} 0.2 \mathrm{mAs}$ & 7 & 2 & 7 & 0 & 1.4 & 0.9 \\
\hline
\end{tabular}

Table 4. Quality image comparison: Speed Class 100 vs Speed Class 400.

\begin{tabular}{|c|c|c|c|c|c|c|}
\hline \multirow{2}{*}{ RX setup } & \multicolumn{2}{|c|}{$\mathrm{N}^{\circ}$ of low contrast inserts detected } & \multicolumn{2}{|c|}{$\mathrm{N}^{\circ}$ of small detail inserts detected } & \multicolumn{2}{|c|}{ Spatial resolution detected $[\mathrm{lp} / \mathrm{mm}]$} \\
\hline & S 100 & S 400 & S 100 & S 400 & S 100 & S 400 \\
\hline $55 \mathrm{kV} 3.2 \mathrm{mAs}$ & 11 & 11 & 14 & 13 & 3.15 & 2.8 \\
\hline $55 \mathrm{kV} 3.2 \mathrm{mAs} 4 \mathrm{mmAl}$ & 9 & 11 & 11 & 12 & 2.5 & 2.5 \\
\hline $60 \mathrm{kV} 1 \mathrm{mAs} 4 \mathrm{mmAl}$ & 7 & 10 & 10 & 12 & 2.5 & 2.8 \\
\hline $60 \mathrm{kV} 1 \mathrm{~m}$ As & 11 & 8 & 12 & 9 & 2.8 & 2.5 \\
\hline $62 \mathrm{kV} 0.4 \mathrm{mAs}$ & 9 & 8 & 9 & 8 & 2.5 & 2.24 \\
\hline $60 \mathrm{kV} 0.4 \mathrm{mAs}$ & 8 & 9 & 9 & 11 & 2.24 & 2.24 \\
\hline $60 \mathrm{kV} 0.32 \mathrm{mAs}$ & 7 & 8 & 8 & 9 & 2.24 & 2.24 \\
\hline $60 \mathrm{kV} 0.2 \mathrm{mAs}$ & 7 & 8 & 7 & 8 & 1.4 & 2 \\
\hline
\end{tabular}

the canopy, using the cassette-holder, and with X-ray tube and NICU at their maximum extension; in this configuration, focus-to-CR distance is compliant with suggestions from EUR16261.

Our study on optimization of exposure parameters showed the impossibility, with our X-ray portable equipment, to lower exposure times below 4 ms as suggested by EUR16261, due to the noticeable degradation on image quality; additional filtration was also shown to be detrimental to spatial resolution and low contrast, and thus discarded.

The workflow we defined involves the selection of $60 \mathrm{kV}, 1 \mathrm{mAs}$ and $6 \mathrm{~ms}$, and it leads to an ESD of approximately $30 \mu \mathrm{Gy}$, which is way lower than the $80 \mu \mathrm{Gy}$ suggested by EUR16261, the $50 \mu \mathrm{Gy}$ suggested by NRPB and the ESD which was imparted before our optimization.

It can be concluded that the delivered dose was reduced by a factor varying from a minimum of $37 \%$ to a maximum of $67 \%$, depending on the operating mode which employed the Technologists before the present study.

Radiation induced risk due to exposure is now approximately at 2.5 cases per million, a value which is negligible compared to the exposures per year performed by the Neonatal Intensive Care of ASLAT-Ospedale Cardinal Massaia.

This study has also made it possible to reduce the risk of induction of tumors, by a factor varying between $60 \%$ and $25 \%$ depending on the operating mode previously used by the Technologists.

Finally, the attempt to optimize the processing mode of the CR led to confirm as optimal the speed class 100, recommended by Agfa and already in use in clinical practice.

\section{References}

[1] Samei, E. and Flynn, M.J. (2002) An Experimental Comparison of Detector Performance for Computed Radiography Systems. Medical Physics, 29, 447-459. http://dx.doi.org/10.1118/1.1449873

[2] Donadieu, J., Zeghnoun, A., Roudier, C., Maccia, C., Pirard, P., Andre, C., et al., (2006) Cumulative Effective Doses Delivered by Radiographs to Preterm Infants in a Neonatal Intensive Care Unit. Pediatrics, 117, 882-888. 
http://dx.doi.org/10.1542/peds.2005-0817

[3] Faulkner, K., Barry, J.L. and Smalley, P. (1989) Radiation Dose to Neonates on Special Care Baby Unit. The British Journal of Radiology, 62, 230-233. http://dx.doi.org/10.1259/0007-1285-62-735-230

[4] Armpilia, C.I., Fife, I.A.J. and Croasdale, P.L. (2002) Radiation Dose Quantities and Risk in Neonates in a Special Care Baby Unit. The British Journal of Radiology, 75, 590-595.

[5] Dougeni, E.D., Delis, H.B., Karatza, A.A., Kalogeropoulou, C.P., Skiadopoulos, S.G., Mantagos, S.P. and Panayiotakis, G.S. (2007) Dose and Image Quality Optimization in Neonatal Radiography. The British Journal of Radiology, 80, 807-815. http://dx.doi.org/10.1259/bjr/77948690

[6] Slade, D., Harrison, S., Morros, S., Alfaham, M., Davis, P., Guildea, Z. and Tuthill, D. (2005) Neonates Do Not Need to Be Handled for Radiography. Pediatric Radiology, 35, 608-611. http://dx.doi.org/10.1007/s00247-005-1414-X

[7] International Commission on Radiological Protection (ICRP) (1991) 1990 Recommendation of the International Commission on Radiological Protection. Annals ICRP, Publication 60, 21.

[8] Appleton, M.B. and Stephen, C.R. (1984) Radiation Protection in a Neonatal Intensive Care Unit: A Practical Approach. Radiography, 50, 137-141.

[9] Hart, D., Wall, B.F., Shrimpton, P.C., Bungay, D.R. and Dance, D.R. (2000) Reference Doses and Patient Size in Paediatric Radiology. NRPB-R318, Chilton, UK.

[10] International Atomic Energy Agency (IAEA) (2013) Radiation Protection in Paediatric Radiology. Safety Report Series, No. 71.

[11] International Commission on Radiological Protection (ICRP) (2013) Radiological Protection in Paediatric Diagnostic and Interventional Radiology. Annals ICRP, Publication 121, 42.

[12] National Radiation Protection Board (NRPB) (2002) Doses to Patients from Medical X-Ray Examinations in the UK. NRPB-W14, Chilton.

[13] European Commission (1996) European Guidelines on Quality Criteria for Diagnostic Radiographic Images in Paediatrics. EUR16261.

[14] Decreto Legislativo (2000) Attuazione della Direttiva 97/43/EURATOM in Materia di Protezione Sanitaria delle Persone Contro i Pericoli delle Radiazioni Ionizzanti Connesse ad Esposizioni Mediche. Gazzetta Ufficiale, Supplement, No. 157.

[15] Puch-Kapst, K., Juran, R., Stoever, B. and Wauer, R.R. (2009) Radiation Exposure in 212 Very Low and Extremely Low Birth Weight Infants. Pediatrics, 124, 1556-1564. http://dx.doi.org/10.1542/peds.2008-1028

[16] Mutch, S.J. and Wentworth, S.D.P. (2007) Imaging the Neonate in the Incubator: An Investigation of the Technical, Radiological and Nursing Issues. The British Journal of Radiology, 80, 902-910. http://dx.doi.org/10.1259/bjr/88577258

[17] Ono, K., Akahane, K., Aota, T., Hada, M., Takano, Y., Kai, M. and Kusama, T. (2003) Neonatal Doses from X-Ray Examinations by Birth Weight in a Neonatal Intensive Care Unit. Radiation Protection Dosimetry, 103, 155-162. http://dx.doi.org/10.1093/oxfordjournals.rpd.a006127

[18] McParland, B.J., Gorka, W., Lee, R., Lewell, D.B. and Omojola, M.F. (1996) Radiology in Neonatal Intensive Care Unit: Dose Reduction and Image Quality. The British Journal of Radiology, 69, 929-937. http://dx.doi.org/10.1259/0007-1285-69-826-929

[19] Brindhaban, A. and Al-Khalifah, K. (2004) Radiation Dose to Premature Infants in Neonatal Intensive Care Units in Kuwait. Radiation Protection Dosimetry, 111, 275-281. http://dx.doi.org/10.1093/rpd/nch338

[20] Frayre, A.S., Torres, P., Gaona, E., Rivera, T., Franco, J. and Molina, N. (2012) Radiation Dose Reduction in a Neonatal Intensive Care Unit in Computed Radiography. Applied Radiation and Isotopes, 71, 57-60. http://dx.doi.org/10.1016/j.apradiso.2012.04.015

[21] Olgar, T., Onal, E., Bor, D., Okumus, N., Atalay, Y., Turkyilmaz, C., Ergenekon, E. and Koc, E. (2008) Radiation Exposure to Premature Infants in a Neonatal Intensive Care Unit in Turkey. Korean Journal Radiology, 9, 416-419. http://dx.doi.org/10.3348/kjr.2008.9.5.416

[22] Wraith, C.M., Martin, C.J., Stockdale, E.J.N., McDonald, S. and Farquhar, B. (1995) An Investigation into Techniques for Reducing Doses from Neo-Natal Radiographic Examinations. The British Journal of Radiology, 68, 1074-1082. http://dx.doi.org/10.1259/0007-1285-68-814-1074

[23] Yu, C.C. (2010) Radiation Safety in Neonatal Intensive Care Unit: Too Little or too Much Concern? Pediatrics \& Neonatology, 51, 311-319. http://dx.doi.org/10.1016/S1875-9572(10)60061-7

[24] Duggan, L., Warren-Forward, H., Smith, T. and Karon, T. (2003) Investigation of Dose Reduction in Neonatal Radiography Using Specially Designed Phantoms and LiF:Mg, Cu, P TLDs. The British Journal of Radiology, 76, 232-237. http://dx.doi.org/10.1259/bjr/79291075 
[25] Jones, N.F., Palarm, T.W. and Negus, I.S. (2001) Neonatal Chest and Abdominal Radiation Dosimetry: A Comparison of Two Radiographic Techniques. The British Journal of Radiology, 74, 920-925.

[26] Hufton, A.P., Doyle, S.M. and Certy, H.M.L. (1998) Digital Radiography in Paediatrics: Radiation Dose Considerations and Magnitude of Possible Dose Reduction. The British Journal of Radiology, 71, 186-199.

[27] Attix, F.H. (1986) Introduction to Radiological Physics and Radiation Dosimetry. John Wiley \& Sons Ltd, New York, 20-34. http://dx.doi.org/10.1002/9783527617135

[28] Tapiovaara, M. and Siiskonen, T. (2008) PCXMC 2.0. User’s Guide. STUK-TR 7, Helsinki.

[29] International Commission on Radiological Protection (ICRP) (2007) Recommendations of the International Commission on Radiological Protection. Annals ICRP, Publication 103, 37. 